\title{
Interstitial lung disease
}

\author{
Katerina M. Antoniou', George A. Margaritopoulos', Sara Tomassetti², \\ Francesco Bonella ${ }^{3}$, Ulrich Costabel ${ }^{3}$ and Venerino Poletti ${ }^{2}$
}

Affiliations: 'Dept of Thoracic Medicine and Laboratory of Cellular and Molecular Pneumonology, Medical School, University of Crete, Crete, Greece. ${ }^{2}$ Pulmonary Unit, GB Morgagni Hospital, Forli, Italy. ${ }^{3}$ Dept of Pneumology and Allergy, Ruhrlandklinik, University Hospital, University of Duisburg Essen, Essen, Germany.

Correspondence: K.M. Antoniou, Dept of Thoracic Medicine and Laboratory of Cellular and Molecular Pneumonology, Medical School, University of Crete, Heraklion 71110 Crete, Greece.

E-mail: kantoniouamed.uoc.gr

ABSTRACT Interstitial lung diseases are a group of diffuse parenchymal lung disorders associated with substantial morbidity and mortality. Knowledge achieved in recent years has resulted in the publication of the new classification of idiopathic interstitial pneumonias, according to which there are three groups: major, rare and unclassified. The novelty of the new classification comes from the fact that difficult to classify entities can be treated according to the disease behaviour classification. Idiopathic pulmonary fibrosis is the most lethal amongst the interstitial lung diseases and presents high heterogeneity in clinical behaviour. A number of biomarkers have been proposed in order to predict the course of the disease and group patients with the same characteristics in clinical trials. Early diagnosis and disease stratification is also important in the field of other interstitial lung diseases.

0 @ERSpublications

A concise review of the progress achieved in interstitial lung diseases during the year 2013 http://ow.ly/sE4MQ

\section{Introduction}

This review reports the relevant progress in the field of interstitial lung disease (ILD) (table 1), as reviewed during the Clinical Year in Review session that was presented at the 2013 European Respiratory Society (ERS) Annual Congress in Barcelona, Spain.

All relevant articles were identified via PubMed using the keywords "interstitial lung disease", "pulmonary fibrosis", "connective tissue disease", and "collagen vascular disease". Articles that were published before the 2012 ERS Annual Congress and after the 2013 ERS Congress were excluded.

\section{Update of the international multidisciplinary classification of idiopathic interstitial pneumonias}

The objective of the 2013 American Thoracic Society (ATS)/ERS classification statement [1] is to update the 2002 ATS/ERS classification of idiopathic interstitial pneumonias (IIPs) (table 2) [2]. Major revisions of the previous classification are summarised below. Cryptogenic fibrosing alveolitis has been removed, leaving the term idiopathic pulmonary fibrosis (IPF). Nonspecific interstitial pneumonia (NSIP) is now accepted as a distinct clinical entity. Major IIPs (e.g. IPF, idiopathic NSIP, respiratory bronchiolitis (RB)-ILD, desquamative interstitial pneumonia (DIP), cryptogenic organising pneumonia (COP) and acute interstitial pneumonia) have been retained, but are distinguished from rare IIPs and unclassifiable cases and grouped into chronic fibrosing (IPF and NSIP), smoking-related (RB-ILD and DIP), and acute/subacute IIPs (COP and acute interstitial pneumonia). Lymphoid interstitial pneumonia (LIP) frequently presents in the context

Received: Dec 132013 | Accepted after revision: Jan 142014

Conflict of interest: None declared.

Provenance: Submitted article, peer reviewed.

Copyright OERS 2014. ERR articles are open access and distributed under the terms of the Creative Commons Attribution Non-Commercial Licence 3.0. 


\title{
TABLE 1 Progress made in the field of interstitial lung disease
}

\section{Key messages}

According to the new classification, IIPs are categorised as major, rare and unclassifiable

MDD discussion is the "gold standard" for the diagnosis of the IIPs

For entities with heterogeneity in the clinical course and that are difficult to classify, a disease behaviour classification complementary to IIP classification has been proposed

IPF is a progressive and lethal fibrosing lung disease with an unpredictable course

Disease stratification aims to identify subgroups of patients who present similar characteristics and should be treated accordingly or enrolled in treatment trials

Gene polymorphisms related to host defence and cell repair are associated with the development of IPF

Markers of IPF progression have been identified by blood transcriptomic and proteomic studies

A combination of blood transcriptome with demographic and functional characteristics provides better information on the outcome of IPF

Early diagnosis and treatment of IPF comorbidities may have a positive effect on patients quality of life

Disease stratification and early identification of patients more likely to progress, and thus need more aggressive treatment, is also important in CTD-ILD

Predicting the clinical course of sarcoidosis, especially lung fibrosis and cardiac and neurological involvement, is still challenging

Potential novel genomic biomarkers, such as multi-gene signatures, can be useful to predict complicated sarcoidosis

In the presence of cardiac sarcoidosis, ICD therapy may be used as a component of an aggressive primary prevention strategy, especially in those patients with ventricular dysfunction

As second-line therapy in sarcoidosis, methotrexate and azathioprine are equally effective in terms of pulmonary function improvement and have a significant steroid-sparing effect

IIP: idiopathic interstitial pneumonia; MDD: multidisciplinary diagnosis; IPF: idiopathic pulmonary fibrosis; CTD: connective tissue disease; ILD: interstitial lung disease; ICD: implantable cardioverter defibrillator.

of other diseases and rarely in its idiopathic form. Thus, this entity has now been moved from the major IIPs to the rare IIPs. In the same group idiopathic pleuroparenchymal fibroelastosis (PPFE) has been added. This entity is characterised by the presence of pleural and subpleural lung parenchyma fibrosis. Two new rare histological patterns of acute fibrinous and organising pneumonia and interstitial pneumonias with a bronchiolocentric distribution have been recognised. A clinical disease behaviour classification has been proposed and molecular and genetic features are being reviewed.

The guideline underlines the importance of multidisciplinary diagnosis (MDD). Adequate presentation and discussion of clinical and radiological data are essential for an accurate MDD. MDD defines the settings in which surgical lung biopsy is required. Observer agreement in the diagnosis of IIPs has been improved substantially due to accumulated experience [3]. In addition, academic physicians in a multidisciplinary setting have shown better diagnostic agreement than community physicians [4]. Based on these findings, current guidelines underline the need for patient evaluation in referral centres using multidisciplinary evaluation [4, 5]. RYERSON and COLLARD [6] have thoroughly discussed the merits and limitations of each possible approach to ILD classification. Despite its recommended use, the multidisciplinary approach has some limitations. First, it is not feasible to implement it in many practice settings. Secondly, it is difficult to standardise as it is subjective and imprecise and, on occasion, results in a diagnosis based on the "most likely" aetiology in the opinion of the "majority" of participants. Finally, there is no clear method to assess the accuracy of these MDD and, assuming agreement is a surrogate of accuracy, it is probably an imperfect surrogate. An aetiology-based classification of ILD appears to be very useful for diseases that are managed by removing the underlying cause or treating the underlying cause directly. However, it fails to identify

\section{TABLE 2 Update of the classification of idiopathic interstitial pneumonias}

\author{
Major idiopathic interstitial pneumonias \\ Idiopathic pulmonary fibrosis \\ Idiopathic nonspecific interstitial pneumonia \\ Respiratory bronchiolitis-interstitial lung disease \\ Desquamative interstitial pneumonia \\ Cryptogenic organising pneumonia \\ Acute interstitial pneumonia \\ Rare idiopathic interstitial pneumonias \\ Idiopathic lymphoid interstitial pneumonia \\ Idiopathic pleuroparenchymal fibroelastosis \\ Unclassifiable idiopathic interstitial pneumonias
}


idiopathic ILD. Another complementary approach to ILD classification is based on disease behaviour. Key advantages are that it is often easier for clinicians and acknowledges diseases heterogeneity, whereas the main limit is that it ignores potentially important details relating to aetiology, which may be important in the prognosis and management of patients. In the future, a mechanism-based classification may allow us to group individual patients with ILD according to disease pathogenesis, allowing more accurate prognostication and targeted therapy. This is the future of medicine and it will revolutionise our approach to the classification and treatment of patients. Unfortunately it is not currently ready for clinical use.

With regard to difficult differential diagnostic aspects, the current guidelines focus on four main concepts. 1) Clinical, radiological and pathological features of chronic hypersensitivity pneumonitis are now better delineated. However, chronic hypersensitivity pneumonitis remains difficult to diagnosis. Current guidelines underline the importance of MDD for the distinction of chronic hypersensitivity pneumonitis from other IIPs, especially when the exposure is not readily apparent. 2) Cases of NSIP with findings suggesting, but not meeting, criteria for a defined CTD are still accepted as an IIP [7]. 3) Familial interstitial pneumonia remains classified as an IIP despite the genetic predisposition. 4) When coexisting patterns occur (NSIP and usual interstitial pneumonia (UIP) or different patterns of smoking-related IIPs), MDD may determine the clinical significance of individual patterns. Combined pulmonary fibrosis and emphysema (CPFE) is an example of coexisting patterns and does not represent a distinctive IIP.

Progress made since 2002 is briefly reported below. The diagnosis of IPF, according to updated evidencebased guidelines [5], requires exclusion of other known causes of ILD, the presence of a UIP pattern on high-resolution computed tomography (HRCT) in patients not subjected to surgical lung biopsy (SLB), and specific combinations of HRCT and SLB patterns in patients subjected to SLB. Patients with IPF and definite UIP on HRCT have a shorter survival than those with indeterminate HRCT findings [8-10]. NSIP is now accepted as a separate entity and criteria were defined in a recent ATS workshop [11]. Recent findings suggest that subpleural sparing may be helpful in distinguishing NSIP from UIP [10]. Consolidation, if present, reflects an organising pneumonia component and may suggest CTD; honeycombing is sparse or absent at presentation but may increase in prevalence and extent during follow-up [11]. The prognosis is variable as some patients improve while others remain stable, but some evolve to end-stage fibrosis and eventually die [11, 12]. The term "smoking-related ILD" has increasingly been used, encompassing most cases of DIP and nearly all cases of RB-ILD and Langerhans' cell histiocytosis [13]. Despite the link with smoking, these diseases remain classified in the IIPs. RB-ILD is increasingly being diagnosed without SLB in smokers with these HRCT findings, and where bronchoalveolar lavage (BAL) demonstrates smokers' macrophages and the absence of lymphocytosis. DIP has been recognised in nonsmokers [13], perhaps reflecting an extension of childhood DIP into adult life (with the latter often due to surfactant protein gene mutations) [14]. 10-year survival remains at $\sim 70 \%$, with resistance to treatment occurring in a significant minority. DIP and RB-ILD need to be distinguished from smoking-related changes, including respiratory bronchiolitis and airspace enlargement with fibrosis, which represent incidental HRCT and histological findings in smokers and are not regarded as distinct forms of IIP. IIPs may have an acute or subacute presentation. Patients with COP typically present with a subacute illness of a relatively short duration (median $<3$ months). COP continues to be included in the classification of IIP because of its idiopathic nature and the tendency, on occasions, for it to be confused with other forms of IIP, especially when there is progression to fibrosis. Acute interstitial pneumonia is a distinct IIP characterised by rapidly progressive hypoxaemia, mortality of $\geqslant 50 \%$ and no proven treatment. HRCT scoring of abnormality extent is independently associated with mortality [15]. Acute interstitial pneumonia can progress to a pattern similar to fibrotic NSIP or to severe fibrosis resembling honeycombing [16]. Acute exacerbation of IIP mostly occurs in IPF, but is also found in other fibrosing interstitial pneumonias [17-23]. Most commonly, the pathology shows a mixed pattern of UIP and diffuse alveolar damage, but organising pneumonia and prominent fibroblastic foci are also described as the acute component [20].

A new category of rare IIPs was created to include idiopathic LIP and idiopathic PPFE. In addition, several rare histological patterns of ILD have been described, including acute fibrinous and organising pneumonia, as well as a group of bronchiolocentric patterns, although these are not recognised as distinct IIPs. Most cases of LIP are associated with other conditions [24], and many of the cases previously diagnosed as LIP are now considered to be cellular NSIP $[2,11]$; therefore, idiopathic LIP has been moved into the category of rare IIPs. Idiopathic PPFE is a rare condition that consists of fibrosis involving the pleural and subpleural lung parenchyma, predominantly in the upper lobes [25]. Acute fibrinous and organising pneumonia was first reported in 17 patients with acute respiratory failure and was initially regarded as a possible new IIP; it has now been classified as a rare histological pattern.

The 2002 ATS/ERS classification proposed an "unclassifiable" category of IIP, acknowledging that a final diagnosis may not be achieved, even after lengthy MDD. If ILD is difficult or impossible to classify, 
management should be based on the most probable diagnosis after MDD and consideration of the expected disease behaviour. Patterns of disease behaviour can be broadly subdivided into: reversible and self-limited (e.g. many cases of RB-ILD); reversible with risk of progression (e.g. cellular NSIP and some fibrotic NSIP, DIP and COP); stable with residual disease (e.g. some fibrotic NSIP); progressive, irreversible disease with potential for stabilisation (e.g. some fibrotic NSIP); and progressive, irreversible disease despite therapy (e.g. IPF and some fibrotic NSIP).

\section{Advances in understanding the pathogenetic mechanisms of IPF}

Despite remarkable improvement in the understanding of IPF, key pathogenetic pathways need to be explored further. The interaction between environmental stressors and genetic predisposition seems to be the key to unlocking and activating the multiple pathogenetic pathways that drive the development of fibrosis [26, 27]. After alveolar epithelium injury there is increased activity of type II alveolar epithelial cells, which proliferate in order to repair the damage. However, the repair process fails and leads to fibrosis. In the fibrotic lung it was observed that angiotensin converting enzyme-2, which showed a protective effect in bleomycin-induced fibrosis [28], is downregulated or absent in actively proliferating epithelial cells and presents a cell-cycle dependent regulation via a JNK mechanism [29].

CHILOSI and co-workers $[30,31]$ speculate that IPF can be related to an acceleration of the ageing process, where the individual genetic background, as well as exposure to a variety of toxic substances, can accelerate pulmonary senescence. Premature ageing and mechanical stress can impair lung function in different ways, affecting distinct progenitor cells and leading to stem cell exhaustion [32]. Moreover CARLONI et al. [33] showed that mechanical stress may play an important role in triggering specific pathways at the periphery of the lung, providing a possible explanation for the peculiar distribution of lung fibrosis in IPF. To further support the concept that IPF is a disease of premature ageing, dehydroepiandrosterone (DHEA) and its sulfated form (DHEA-S), which have been previously linked to the impaired function of the immune system occurring with ageing, have been evaluated in the serum of IPF patients [34]. The levels of both steroids were decreased in males with IPF whereas only DHEA-S was reduced in females with IPF. Moreover, the effect of DHEA has been investigated in cell cultures of normal human lung fibroblasts and it has been observed that this steroid decreases fibroblast proliferation and increases apoptosis. The effect in other steps of the fibrogenetic process is also important as the authors showed that DHEA reduces the fibroblast to myofibroblast differentiation and the collagen production induced by transforming growth factor (TGF)- $\beta 1$, as well as fibroblast migration induced by platelet-derived growth factor. Because of these multiple antifibrotic properties, DHEA could represent an intriguing future therapeutic option.

Studies published recently regarding genetic phenotyping, peripheral blood transcriptome, and plasma and serum biomarkers have tried to shed light on what seems to be the main target in future IPF research in terms of treatment, namely disease stratification [35, 36]. Recently, polymorphisms in several genes associated with the development of either sporadic or familial forms of IPF have been investigated. A common polymorphism in the promoter region of the $M U C 5 B$ gene has been estimated to increase the risk of IPF six-fold for heterozygotes and 20-fold for homozygotes [37]. MUC5B is a mucin secreted in the airways and implicated in mucosal immune defence by protecting the surface epithelium of the airways, properties that make its role in the development of an ILD, such as IPF, unexpected. It has been suggested that increased secretion of MUC5B may interfere with the normal repair process of the epithelium or may be directly toxic and stimulate a fibroproliferative response. However, it should be stressed that $19 \%$ of the unaffected patients also have the MUC5B risk variant, suggesting that other genetic variants may contribute to the development of IPF in combination with the MUC5B risk variant. Subsequently, and somewhat surprisingly, it was observed that this polymorphism conferred a survival benefit in IPF patients [38], and that it was associated with a trend towards slower decline of forced vital capacity (FVC) suggesting the presence of at least two different clinical subtypes based on this signal [39]. Hypotheses for this apparently contradictory finding include an enhanced mucosal defence with protection against infection and a potential dual role of $M U C 5 B$ in wound healing. Two studies have shown that this polymorphism is associated with an IPF-specific pathway by confirming previous findings and demonstrating no association with the development of fibrosis in the context of sarcoidosis and systemic sclerosis (SSc) [39, 40]. Other evidence that distal airways may participate in the development of IPF has been recently suggested [41]. In lung tissues of IPF patients, cilium-associated genes were expressed and, importantly, the higher expression was accompanied by increased expression of $M U C 5 B$ and matrix metalloproteinases (MMP)-7 which identified a distinct clinical phenotype characterised by more extensive microcystic honeycombing and possibly worst outcome. This finding was further validated in an independent cohort of IPF patients.

A genome-wide association study of 2492 individuals with fibrotic IIPs and 6573 controls has also confirmed previous findings that risk variants in three loci on the telomerase RNA component, telomerase reverse transcriptase and MUC5B genes are associated with the development of IIPs [37, 42, 43] and, in 
addition, has identified seven more loci related to genes controlling host-defence, cell-cell adhesion and DNA repair suggesting that alterations of these mechanisms may promote fibrogenesis [44]. Another genome-wide association study of 1428 IPF patients and 1931 controls has identified single nucleotide polymorphisms (SNPs) in the gene for TOLLIP and SPPL2C that also promote fibrogenesis [45]. However, a contradictory finding that merits further investigation has been observed. On the one hand, the specific minor allele rs 5743890 protects against fibrosis and, on the other hand, is associated with more rapid disease progression and mortality.

The peripheral blood transcriptome can be used in order to identify the presence of IPF and importantly correlates well with disease severity. 1428 genes were differentially expressed in patients characterised as having mild IPF (diffusing capacity of the lung for carbon monoxide (DLCO) $<60 \%$ predicted) compared to controls and in 2790 with severe IPF (DLCO $<35 \%$ pred) compared to controls, whereas 13 genes were differentially expressed between patients with mild and severe disease [46]. Most of these genes are implicated in the function of the immune system and host defence. A recent study has added more useful information about the ability of the transcriptome to predict outcome [47]. Using two independent cohorts, the authors examined peripheral blood mononuclear cell gene expression and found 52 genes associated with transplant-free survival (TFS). The genes CD28, ICOS, LCK and ITK were under expressed and associated with shorter TFS. The gene expression, evaluated with quantitative RT-PCR, was decreased in IPF and when combined with demographic (age and sex) and functional (FVC) characteristics provided a better outcome prediction.

Previous findings that peripheral blood proteins, such as mucin-1 (MUC1-KL6), CC chemokine ligand-18 (CCL-18) and surfactant protein-A [48-50], are related to increased mortality have been enhanced by a large-scale follow-up study which identified that MMP-7 (a marker of alveolar epithelial cell injury), S100A12 and interleukin (IL)-8 (markers of neutrophil recruitment and activation), intercellular adhesion molecule-1 and vascular cell adhesion molecule-1 (markers of oxidative stress in the lung) in both derivation and validation cohorts of IPF patients were predictive of poor survival [51].

Deregulation of humoral immunity is associated with IPF. B-cell aggregates, activated T-cells and mature dendritic cells have been reported in the IPF lung, increasing the likelihood of antigen presentation activity $[52,53]$. Moreover, activated $\mathrm{CD} 4^{+} \mathrm{T}$-cells produce either cytokines, which induce the production of autoantibodies from B-cells, or mediators such as IL-10, TGF- $\beta 1$ and tumour necrosis factor (TNF)- $\alpha$, which promote fibrogenesis [54]. For instance, the presence of auto-antibodies against periplakin, a small protein localised in desmosomes, in the serum and BAL of IPF patients was associated with severe disease [55]. Antibodies against heat-shock protein (HSP)-70 have been observed in 25\% of 122 patients with IPF and were present in $70 \%$ of the patients who experienced acute exacerbation. In terms of disease progression, anti-HSP-70 was associated with greater FVC reduction and reduced 1-year survival [56]. The study leaves the option open for the future use of drugs targeting humoral dysregulation, such as rituximab, after the disappointment caused by the results of the PANTHER-IPF study [57], despite criticisms about their interpretation [58].

Innate immunity has been proposed to have a role in the development and progression of IPF, mainly through the activation of Toll-like receptor (TLR)-9 [59, 60]. TLR-3, amongst other properties, regulates the recognition of viral pathogen-associated molecular patterns. Viruses have been implicated in both pathogenesis and acute exacerbations of IPF $[61,62]$. The TLR-3 L412F polymorphism has been associated with defective function of the receptor and with accelerated disease progression [63].

In the past few years there has been a lot of discussion about the necessity of the early identification and treatment of various comorbidities in IPF and how this can improve the quality of life of the patients [26]. Pulmonary hypertension is a well-recognised comorbidity in ILDs and is considered as a predictor of poor survival and an indicator for immediate listing for lung transplantation [64]. Recently, it was confirmed that pulmonary hypertension has an important impact on outcome as, in a cohort of patients with advanced IPF assessed for lung transplantation, the presence of pulmonary hypertension at baseline was associated with a significantly higher risk for the development of adverse events and, consequently, with poor survival [65]. The gene expression profile of laser captured microdissected pulmonary arterioles was compared between patients with IPF-associated pulmonary hypertension, IPF without pulmonary hypertension and controls. The vascular gene expression signal, which was characterised by aberrant cellular growth and altered apoptosis, was different in IPF patients compared to controls, but was similar in two IPF groups suggesting that preclinical vascular disease may be present in patients with normal tests during work-up for the diagnosis of pulmonary hypertension [66]. 


\section{Adding pieces to the complex IPF clinical profile puzzle}

IPF is a chronic, fibrosing ILD characterised by heterogeneous clinical features and a highly variable course with inter-individual variability that impairs our ability to predict prognosis. The clinical spectrum of this disease, and its morbidity and mortality, are also influenced by the coexistence of multiple comorbidities that are now better recognised, including gastro-oesophageal reflux disease, venous thromboembolism, coronary artery disease, sleep disordered breathing, depression, emphysema, pulmonary hypertension and lung cancer. The identification and treatment of comorbidities may improve morbidity and potentially impact on mortality, thus awareness of the spectrum of comorbidities is important in optimising outcomes in this group of patients [67]; especially for gastro-oesophageal reflux disease (GORD), infections and pulmonary hypertension, which seem to impact on disease progression and increase the risk of an acute exacerbation of IPF [68].

AlLAIX et al. [69] compared the clinical presentation, the oesophageal function and the reflux profile in 80 patients with GORD to 22 patients with GORD and IPF. Results showed that GORD is often asymptomatic in IPF patients, with heartburn present in $<60 \%$ of patients with GORD and IPF. The upper oesophageal sphincter was more frequently hypotensive in IPF patients, with a higher proximal oesophageal acid exposure and a slower supine acid clearance in patients with GORD and IPF compared with patients with GORD only. Reflux features have been further investigated by SAVARINO et al. [70]. 40 consecutive patients with IPF, 40 patients with ILD other than IPF and 50 healthy volunteers were studied. IPF patients had significantly higher oesophageal acid exposure (9.25\% versus $3.3 \%$ versus $0.7 \%$ ) and proximal reflux events (median 51 versus 20 versus nine) compared to non-IPF patients and healthy volunteers, respectively. Pulmonary fibrosis HRCT scores correlated well with reflux episodes. These studies show that IPF patients had a very severe degree of overall reflux disease compared, not only with non-IPF patients, but also with those with other respiratory disorders with an established GORD association, such as asthma, cough and laryngitis. Pulmonary fibrosis patients have a high risk of pulmonary aspiration of gastric contents even in the absence of typical reflux symptoms, and despite evidence of normal oesophageal peristalsis. The increased frequency of reflux implies that GORD should be documented and treated adequately to prevent microaspiration of gastric contents and its potential deleterious effect in the induction, progression and/or exacerbation of pulmonary fibrosis in patients with IPF.

Emphysema can be associated with IPF as described in CPFE syndrome by COTTIN and co-workers [71, 72]. A recent study by RYERSON et al. [73] revised this entity and attempted to better define its clinical profile and prognosis. Clinical characteristics and outcomes of CPFE and non-CPFE IPF patients were compared in two large cohorts of 365 IPF patients. 29\% of patients had some emphysema detectable on HRCT, $13.4 \%$ had at least $5 \%$ emphysema. The authors choose a pre-specified cut-off of $\geqslant 10 \%$ emphysema to define CPFE based on its ease of use, excellent inter-rate reliability, and its potential clinical relevance in identifying Global Initiative for Chronic Obstructive Lung Disease (GOLD) grade II equivalent disease. CPFE defined according to these criteria was present in $8 \%$ of cases. Overall agreement between the two radiologists on the presence of CPFE was $96 \%$ ( $\kappa$-value of 0.74 ), and did not change significantly for different threshold definitions. Patients with CPFE had more extensive smoking history, greater oxygen requirements, higher pulmonary artery pressure, less restrictive physiology and lower diffusing capacity. Patients with IPF and CPFE have less physiological restriction and worse gas exchange compared to IPF patients without emphysema. The higher FVC found in patients with CPFE is only partially explained by less severe fibrosis in these patients, as illustrated by the difference in FVC that remains after adjustment for fibrosis score. It seems most likely that emphysema is mitigating the impact of the fibrosis on ventilatory physiology. Fibrosis and emphysema have similar and cumulative effects on gas exchange, reflected by disproportionate oxygen requirement, low DLCO and pulmonary hypertension in CPFE. Inhaled therapies for chronic obstructive pulmonary disease (COPD) were used in 53\% of patients with CPFE. By using a 10\% threshold to define CPFE, which probably corresponds to GOLD grade II disease or worse, the authors identified a population of patients who might benefit from short- and long-acting bronchodilators, with the addition of inhaled corticosteroids in some patients. There was no association between the diagnosis of CPFE and disease progression on unadjusted or adjusted analyses. The two groups of patients had similar mortality and the same median TFS of 2.8 years. These data are in contrast with previous studies that suggested that CPFE patients have a worse prognosis compared to non-CPFE patients [71, 72]. One remaining question is whether CPFE represents a biologically distinct condition (i.e. a different disease) or is just IPF and emphysema in the same patient. As claimed by CHILOSI and co-workers [30, 31], different pulmonary diseases, including COPD/emphysema and IPF, can be related to an acceleration of the ageing process. The individual genetic background, as well as exposure to a variety of toxic substances (primarily cigarette smoke), can contribute significantly to accelerating pulmonary senescence. Premature ageing and mechanical stress can impair lung function in different ways, affecting distinct progenitor cells (mesenchymal stem cells in COPD and alveolar epithelial precursors in IPF) and leading to stem cell 
exhaustion [32]. Future research in CPFE will require evaluation of underlying biological pathways to address this question and explain why some smokers develop emphysema, some IPF and some CPFE.

It has been shown that IPF patients are more likely than controls to have venous thromboembolism [74], and both epidemiological and laboratory studies suggest that activation of the coagulation cascade within the lung may be involved in the pathogenesis of IPF. BARGAGLI et al. [75] showed procoagulant status to correlate with IPF and acute exacerbation of IPF compared to both NSIP and healthy controls. NAVARATNAM et al. [76] investigated the presence of a pro-thrombotic state in a large cohort of patients. They recruited 211 incident cases of IPF and 256 age- and sex-matched general population controls and reported that IPF cases were more than four times more likely than controls to have a pro-thrombotic state (OR 4.78 (95\% CI 2.93-7.80); $\mathrm{p}<0.0001)$. Cases with a pro-thrombotic state were also likely to have more severe disease at presentation (FVC $<70 \%$ pred; OR 10.79 (95\% CI 2.43-47.91)) and had a three-fold increased risk of death (hazard ratio (HR) 3.26 (95\% CI 1.09-9.75)). While the results of these investigations establish a strong association between a pro-thrombotic state and IPF, they do not prove causation and clinical management of patients remains to be further elucidated. Experimental evidence from clinical trials assessing the efficacy of anticoagulants for IPF treatment has provided conflicting results $[77,78]$, and a retrospective study has recently shown that even the current use of an anticoagulant for a cardiovascular indication can worsen IPF progression and survival [79]. These results suggest that biomarkers of coagulation, along with other 'omics-based biomarkers, may be useful to identify high-risk individuals and to target investigations of primary and secondary prevention. However, future research needs to focus on novel agents for prevention [80].

The prevalence of pulmonary hypertension in the setting of IPF is reported to be $32-85 \%$, and CPFE has been recognised as a strong determinant of secondary pre-capillary pulmonary hypertension [68, 81]. Recent evidence reinforces the view that pulmonary hypertension is an important contributor to increased mortality and morbidity in IPF patients [82, 83], and has recognised the need to diagnose the coexistence of pulmonary hypertension early in order to evaluate selected patients for lung transplant in a timely manner. Despite the fact that medications currently approved to treat pulmonary hypertension have been administered for pulmonary hypertension in the setting of IPF, reliable data on the safety and efficacy of pulmonary hypertension treatment are lacking. RAGHU et al. [84] conducted a large randomised, doubleblind, placebo-controlled trial (www.ClinicalTrials.gov identifier NCT00768300) which was designed to test the safety and efficacy of ambrisentan in reducing the rate of IPF progression. The trail was terminated early after the enrolment of 492 patients (75\% of intended enrolment) because ambrisentan-treated patients were more likely to experience disease progression (90 (27.4\%) versus $28(17.2 \%)$ patients, HR 1.74 (95\% CI $1.14-2.66) ; \mathrm{p}=0.010)$. Respiratory hospitalisations were seen in $44(13.4 \%)$ and nine $(5.5 \%)$ patients in the ambrisentan and placebo groups, respectively $(\mathrm{p}=0.007) .26(7.9 \%)$ patients who received ambrisentan and six $(3.7 \%)$ who received placebo died $(\mathrm{p}=0.100) .32(10 \%)$ ambrisentan-treated patients and $16(10 \%)$ placebo-treated patients had pulmonary hypertension at baseline, and analysis stratified by the presence of pulmonary hypertension revealed similar results for the primary end-point. To date, pulmonary hypertension remains a poorly understood comorbidity in IPF that significantly impacts on prognosis, but it is still very challenging to treat.

Infectious agents, including both viruses and bacteria, have been associated with IPF and may influence disease behaviour $[58,85,86]$. If infections play a causal role in disease progression and acute exacerbations it is possible that antiviral and antibiotic drugs may be effective in modifying the course of this devastating disease. Shulgina et al. [87] conducted a double-blind, multicentre study among 181 patients with fibrotic IIP (89\% diagnosed as definite/probable IPF) randomised to receive co-trimoxazole $960 \mathrm{mg}$ twice a day or placebo for 12 months. Co-trimoxazole had no effect on FVC, DLCO, 6-min walk test or Medical Research Council dyspnoea score. However the use of co-trimoxazole reduced respiratory tract infections and resulted in a significant reduction in the percentage of patients requiring an increase in oxygen therapy (OR 0.05 (95\% CI $0.00-0.61)$ ) and in all-cause mortality (co-trimoxazole: three out of 53; placebo: 14 out of 65) (HR 0.21 (95\% CI 0.06-0.78); $\mathrm{p}=0.02)$. Compared with placebo the incidence of nausea and rash was increased. The use of co-trimoxazole can't be widely adopted based on these preliminary results, but this trial encourages future investigations in this direction.

Another issue that has been discussed is whether circulating autoantibodies in IPF are clinically meaningful. The role of autoimmunity is better delineated in other ILDs, such as idiopathic NSIP [88], but it is still unclear in IPF. LEE et al. [89] compared 67 IPF patients to 52 healthy controls and found positive autoantibodies in $22 \%$ of IPF patients and $21 \%$ of healthy controls, without significant differences in the types of autoantibodies or clinical characteristics between IPF, with and without circulating autoantibodies. The presence of circulating autoantibodies was associated with longer TFS time on adjusted analysis; however, the significance varied depending on which statistical model was used (HR 0.22-0.47, p-value 0.02-0.17). This result needs to be confirmed by further studies. 
The recent literature shows an increased incidence of obstructive sleep apnoea (OSA) in patients with ILD, and particularly with IPF [90]. MERMIGKIS et al. [91] treated 12 patients with newly diagnosed IPF and moderate-to-severe OSA with continuous positive airway pressure (CPAP), confirmed by overnight attended polysomnography, and found a significant improvement in daily living activities based on the Functional Outcomes in Sleep Questionnaire. Despite intense follow-up, two patients had difficulty complying with CPAP and stopped treatment after the first month.

Many recent studies are focusing on the quality of life issues of IPF patients, and depression is increasingly being recognised as an important comorbidity in IPF [92]. BAJWAH et al. [93] conducted the first study to examine in depth the impact of symptoms and psychosocial needs of IPF patients' and of their informal caregivers'. The authors revealed the profound effect on every aspect of the patients' and caregivers' lives. RAVAGLIA et al. [94] reported on the possible organisational support related to the dying process and quality of care in the last week of life of IPF patients with acute exacerbation who did not respond to treatment. Patients were referred to a palliative care unit with benefit in palliation of symptoms and response to patient's and family needs. Despite a wide acknowledgment that patients with nonmalignant diseases would benefit from access to palliative care, debate continues as to whether or how this can be. To understand this better we need research that addresses the impact of general and specialist palliative care on the physical and psychosocial health of IPF patients and their caregivers.

In the past decade numerous multicentre, randomised, placebo-controlled trials have investigated different agents with different mechanisms of action. Based on some of these studies the first IPF-specific drug, pirfenidone, has recently been licenced for use in Europe [95]. In the past year, safety reports of pirfenidone in real-life practice have been published both from Japan and Europe, confirming that pirfenidone is well tolerated and can improve progression-free survival in IPF [96-98]. However, this is just the first step on a long road towards successful treatment of IPF, and we should keep walking with it clear in our mind that combinations of various agents may be more effective [99]. As has been shown for the treatment of lung cancer with which IPF presents many similarities [100]. Many other therapeutic trials are still ongoing, and the results of large trials with novel agents, such as nintedanib (www.ClinicalTrials.gov identifier NCT01335477) and N-acetylcystine (PANTHER-IPF, www.ClinicalTrials.gov identifier NCT00650091) are due. Other approaches, such as regenerative medicine, are pointing towards a successful application of stem cell treatment in a degenerative disease such as IPF and are showing intriguing preliminary results [101].

\section{ILD associated with collagen tissue disorders}

ILD associated with SSc represents, together with pulmonary hypertension, the most common cause of death [102]. Fibroblasts present altered characteristics in SSc and play an important role in the development of lung fibrosis by producing excessive amounts of extracellular matrix. It is believed that targeting lung fibroblasts may be an important step for future treatment. A recent mRNA microarray study of lung fibroblasts in SSc-associated NSIP has produced important findings [103]. First, a similarity in gene expression profile with IPF has been observed and a drug targeting fibroblasts may be effective in both diseases. However, this finding warrants further study because of the small number of IPF patients. Secondly, the authors observed a suppression of a large gene programme, namely interferon-stimulated genes, in both diseases compared to controls. It can be hypothesised that this suppression could enhance lung fibrosis progression through fibroblast proliferation and apoptosis resistance. Interestingly, suppression of interferon-stimulated genes has also been observed in cancers representing one more similarity between fibrogenesis and tumorigenesis [103].

Disease stratification and early identification of patients who are more prone to progress, and thus in need of more aggressive treatment, is also important in the field of SSc-ILD. In this regard, a staging system using disease extent on HRCT with an ancillary role for the lung function test in "indeterminate" cases [104, 105], and a series of markers of epithelial injury and epithelial cell damage have been implemented [106-109]. Recently, increased levels of IL-6 have been proposed as marker of a decline in FVC and/or DLCO within the first year, and of death within the first 30 months in patients with mild disease [110]. Patients who are in the "grey zone" between mild and extensive disease would either progress at some stage in the future or remain stable. It is the former group that needs to be identified at an early stage in order to receive more aggressive treatment with the aim to prevent progression, whereas in the latter group a policy of masterful inactivity with very close follow-up should be adopted. The authors concluded that IL-6 could be a useful marker for this purpose after validation in prospective studies.

Pulmonary complications in rheumatoid arthritis (RA) are not uncommon and account for the death of $10-20 \%$ of patients. Moreover, it was recently observed that clinically significant ILD associated with RA (RA-ILD) occurs in $10 \%$ of the patients and is associated with shorter survival and more severe underlying disease [111]. 
Interestingly, the subgroup of patients with RA-UIP, which is also the pathological counterpart of IPF, tends to have the same dismal outcome as IPF. Thus, early identification of ILD is crucial.

Citrullination is a post-translational modification, most probably triggered by cigarette smoking. It has been postulated that citrullinated antigens may be neo-epitopes, which break immunological tolerance and lead to RA and other extra-articular manifestations. Anti-citrullinated protein antibodies (ACPA) have been found to precede the diagnosis of RA and two elegant studies have tried to shed light on their involvement in the development of RA-ILD. In the first study [112], methods based on differential immunoprecipitation of citrulinated extracts have identified citrullinated versions of $\mathrm{Hsp} 90 \alpha$ and $\mathrm{Hsp} 90 \beta$ as autoantibody targets distinguishing RA-ILD from RA without ILD, mixed connective tissue disease (CTD) and IPF. These autoantibodies showed low sensitivity (20-30\%), but were highly specific for RA-ILD (>95\%) [112].

In the second study [113], the relationship between the number and fine specificity of ACPA with the presence and extent of RA-ILD on HRCT have been explored. The levels of ACPA were, as expected, higher in smokers compared to nonsmokers in RA-ILD compared to RA whereas the levels of antibodies against non-citrullinated antigens were similar in both subgroups. Moreover, an expanded repertoire of ACPA was an indicator of fibrotic elements in HRCT, such as reticulations, honeycombing and traction bronchiectasis, and of restrictive pattern on lung function tests. In addition, ACPA were more associated with the UIP pattern than with NSIP. RA-UIP presents a worse outcome than RA-NSIP and the findings of this study may, at least partly, explain these differences in pathogenesis and outcome.

An elegant study has provided important and intriguing evidence of a possible pathogenetic linkage between RA-ILD, IPF and smoking-related emphysema. The authors have observed that in both IPF and RA-ILD, the prevalence of emphysema, evaluated on HRCT, was associated with a surprisingly lower pack-years smoking history compared to control patients with or without COPD [114]. Another important finding was that in smokers there was increased prominence of honeycombing and coarseness of fibrosis. Taken together, these findings justify the future evaluation of pathogenetic pathways common to pulmonary fibrosis and smoking-related lung damage.

CTD-ILDs are usually treated with anti-inflammatory and immunosuppressive drugs and most of the time respond well to these drugs. However, discontinuation of the treatment due to side-effects or progression of the disease despite treatment may occur. While the results of the ongoing Scleroderma Lung Study II, which compares the effect of cyclophosphamide versus mycophenolate mofetil, are awaited, a retrospective study of a large number of patients with CTD-ILD has shown that mycophenolate mofetil is well tolerated with low rate of discontinuation when used either as a second- or third-line steroid sparing drug when cyclophosphamide or azathioprine are discontinued because of increased toxicity [115]. Moreover, in patients with CTD-UIP, the use of the drug was associated with stabilisation of both FVC and DLCO, whereas both parameters were significantly improved in non-CTD-UIP patients. The effect of rituximab, a chimeric monoclonal antibody with high affinity for the CD20 surface antigen expressed on pre-Blymphocytes and B-lymphocytes resulting in depletion of B-cells from the peripheral circulation for up to 6-9 months, has been evaluated in a large cohort of patients with non-IPF ILD in which CTD-ILD was the predominant disease pattern [116]. Patients with previously progressive disease despite immunosuppression have experienced an improvement in FVC and at least a stabilisation of DLCO 6-12 months following treatment with rituximab.

\section{Sarcoidosis}

Predicting the clinical course of sarcoidosis, especially the evolution into severe lung dysfunction or cardiac and neurological involvement (complicated sarcoidosis), represents a challenge for physicians dealing with this disease. At present, currently available biomarkers fail to identify individuals at risk for complicated sarcoidosis. In a recent study, ZHOU et al. [117] attempted to discover a universal gene signature to distinguish individuals at risk of sarcoidosis in comparison to healthy controls and patients with other fibrotic lung disorders, and to identify complicated sarcoidosis. For this purpose, the genome-wide geneexpression data of African-American and European descent ancestries were compared. Initially, sarcoidosis candidate gene studies focused on granuloma formation and selected immune response pathways; in particular, those involved in antigen presentation such as the human leukocyte antigen loci [118-120]. In recent years, genome-wide association studies have become more accessible and a German study on 947 sarcoidosis patients found a strong association for a polymorphism in the butyrophilin-like 2 (BTNL2) gene with the development of sarcoidosis [121]. ZHOU et al. [117], using genome-wide peripheral blood-gene expression analysis, identified a non-targeted 20-gene sarcoidosis biomarker signature that was able to distinguish sarcoidosis from healthy controls (accuracy of $86 \%$ ), and which also served as a molecular signature for complicated sarcoidosis (accuracy 81\%). In an elegant fashion, the authors compared this unbiased 20-gene signature with a 31-gene signature targeting the T-cell receptor/JAK-STAT/cytokine-cytokine receptor 
signalling pathway genes implicated in the pathogenesis of sarcoidosis, with the latter achieving lower accuracy in predicting complicated sarcoidosis. These results were validated in two different independent sarcoidosis blood gene expression datasets. Interestingly, 30 SNPs corresponding to six genes of the signature were found to play a possible role in regulating the expression and determining the variation of sarcoidosis signature genes [117]. Despite the small sample size of patients, especially those with complicated sarcoidosis, a potential novel genomic biomarker in the diagnosis of sarcoidosis, as well as for diseases with a complicated course, has been identified. Further validation in a replicate cohort, as well as testing versus other granulomatous disorders, is necessary to confirm the universal role of this 20-gene signature.

Phenotyping sarcoidosis patients is of central importance for targeted management and treatment. For example, patients with pulmonary sarcoidosis in fibrotic stage can have a chronic progressive evolution or can develop acute worsening of respiratory symptoms. The prevalence of acute worsening of lung sarcoidosis remains unknown. In the past this clinical entity has been described as acute pulmonary exacerbations of sarcoidosis [122]. Recently, BAUGHMAN et al. [123] studied 129 patients with fibrotic sarcoidosis. Acute worsening of sarcoidosis, which was defined as an episode in which the patient was either prescribed an antibiotic for 5-21 days and/or a short increase in systemic glucocorticosteroids for increased respiratory symptoms (an abnormal imaging was not mandatory), occurred in $73 \%$ of cases. Patients with bronchiectasis on HRCT, as well as patients receiving anti-TNF therapies, experienced a higher number of acute worsening events [123]. Even if this study has several limitations, above all the retrospective design, the lack of radiological data and the lack of investigation of the effect of steroid dosage on the frequency of acute events, this is the first study that has systematically focused on this aspect. Further studies are necessary to confirm the existence of acute worsening as a separate clinical entity or phenotype; however, what emerges from this study is that patients with fibrotic sarcoidosis should be carefully managed and properly treated in order to reduce the risk of acute worsening events [123].

With regard to recent advances in the diagnostic methods of sarcoidosis, it is still under debate as to whether endobronchial ultrasound-guided transbronchial needle aspiration (EBUS-TBNA) is superior to transbronchial lung biopsy (TBLB) for the diagnosis of sarcoidosis [124].

The usefulness of EBUS-TBNA for diagnosing sarcoidosis was first reported in 2007, in a series of 65 patients where the sensitivity was $92 \%$ [125]. Subsequent studies have suggested that EBUS-TBNA is more sensitive compared to standard TBLB, especially for radiological stage I disease [124, 126]. Recently, vON BARTHELD et al. [127] published the results of a randomised controlled trial (GRANULOMA) comparing conventional bronchoscopy (including transbronchial and endobronchial mucosal biopsies) with endosonography (oesophageal or endobronchial ultrasonography)-guided nodal aspiration for the detection of noncaseating granulomas in patients with suspected pulmonary sarcoidosis. Of 304 randomised patients, 149 were randomised to conventional bronchoscopy with transbronchial and mucosal biopsies and 155 to endosonography. BAL was performed in all patients. The primary outcome was to detect noncaseating granulomas in patients with a final diagnosis of sarcoidosis. Endosonography reached a diagnostic yield of $80 \%$ while TBLB reached only $53 \%$. Among patients with suspected stage I/II pulmonary sarcoidosis, endosonography compared with TBLB resulted in a greater diagnostic yield (84\% and $77 \%$ versus $38 \%$ and $66 \%$ for stage I and II, respectively). These results are in line with previous findings [128]. However, the yield of bronchoscopic biopsy was astonishing low (53\%) compared to previous reports which described yields of $\geqslant 80 \%$ for all sarcoidosis stages. With regard to the safety profile, the secondary end-point of the study, serious adverse events were uncommon with both procedures. Despite several limitations, such as the presence of an incorporation bias, this study is important because it states the importance of endosonographic evaluation over other procedures in confirming sarcoidosis [127].

The evaluation of symptoms and health status in sarcoidosis patients is critical, together with objective parameters, for the decision to start treatment. The King's Sarcoidosis Questionnaire (KSQ), recently proposed by PATEL et al. [129], is a modular multi-organ health status measure for patients with sarcoidosis. It is the first tool to assess organ-specific health status and can be used in clinics and research to aid evaluation of therapies. As described in the study by PATEL et al. [129], a preliminary KSQ was validated by the authors using a systematic statistical approach with Rasch analysis, commonly used in the development of health status tools. Redundant items were removed on the basis of the first analysis. The final questionnaire, tested in 207 patients with sarcoidosis, consists of five modules: general health status (10 items); lung (six items); medication (three items); skin (three items); and eye (seven items). The KSQ correlated with other questionnaires, such as the St George's Respiratory Questionnaire and the Short-Form 36 questionnaire, and correlated weakly with pulmonary function tests. In comparison to the classical Sarcoidosis Health Questionnaire [130], the KSQ has several advantages. 1) It can be tailored to individual clinical phenotypes and contains more items assessing fatigue, medications and extra-pulmonary organ involvement including eye disease. 2) It is a quick, highly repeatable and valid tool to identify health status issues that are important to 
patients in the clinic. Interestingly, a specific medication module can be used to assess the impact of medications on health status. Even if further studies are needed to investigate whether excluded items, such as sleep disturbances and sexual health, could impact on the utility, the KSQ represents an important advance for a more precise cross-sectional and longitudinal assessment of patient's health status.

In the management of sarcoidosis patients, cardiac involvement often represents a pitfall. Cardiac involvement is accurately diagnosed before death in only $29 \%$ of patients but it accounts for $13-25 \%$ of all deaths in patients with sarcoidosis [131]. Sudden death is the most common manifestation of cardiac sarcoidosis, involving $>60 \%$ of patients [132]. It is unclear whether implantable cardioverter defibrillator (ICD) therapy is effective in the prevention of sudden death. In a recent study by SCHULLER et al. [133], 112 subjects with cardiac sarcoidosis were investigated in three institutions. 32\% of the subjects received appropriate therapy for ventricular arrhythmias over the follow-up period and an ICD storm, defined as three or more appropriate ICD therapies in $24 \mathrm{~h}$, occurred in $14 \%$ of the patients. Through multivariate analysis the investigators found that left and right ventricular dysfunction were significant predictors of future ICD therapies (OR 6.52 and 5.64 for left and right ventricular dysfunction, respectively) [133]. Interestingly, even among 83 patients with a primary prevention device, the incidence of appropriate ICD therapies was $11.3 \%$ annually. Based on these findings the authors suggest an aggressive prospective screening approach for sarcoidosis patients in whom cardiac involvement is suspected. When cardiac involvement is established, ICD therapy may be used as a component of an aggressive primary prevention strategy, especially in those patients with ventricular dysfunction.

With regard to sarcoidosis treatment, although new therapies for sarcoidosis are emerging, long-term steroid treatment is still the gold standard [134]. Methotrexate and azathioprine are used in the clinical practice as second-line therapeutics for refractory sarcoidosis or as steroid sparing agents [135]. In a recent study, VORSELAARs et al. [136] investigated the efficacy of methotrexate versus azathioprine as first-choice second-line therapy in sarcoidosis.

In this retrospective study, 145 patients treated with methotrexate and 55 with azathioprine were included. Both medications showed similar positive effects on lung function (improvement in forced expiratory volume in $1 \mathrm{~s}$ and DLCO at 1 year). A significant steroid sparing potency was seen for both drugs, with a decrease of the daily dosing regimen of $6.32 \mathrm{mg} \cdot \mathrm{year}^{-1}$ [136]. Although the results concerning methotrexate confirm previous observations $[137,138]$, this is the first time that comparable efficacy with azathioprine has been reported. With regard to the safety profile, the incidence of side-effects was comparable for both drugs, except there was a higher infection rate in the azathioprine group (35\% versus 18\%). The major concern of this study is that two different institutions, in two different countries, followed two cohorts using one or the other drug in a non-random fashion. However, the study shows that both drugs are equally effective in terms of pulmonary function improvement and have a significant steroid-sparing effect [136].

\section{References}

1 Travis WD, Costabel U, Hansell DM, et al. An official American Thoracic Society/European Respiratory Society statement: update of the international multidisciplinary classification of the idiopathic interstitial pneumonias. Am J Respir Crit Care Med 2013; 188: 733-748.

2 American Thoracic Society, European Respiratory Society. American Thoracic Society/European Respiratory Society international multidisciplinary consensus classification of the idiopathic interstitial pneumonias. Am J Respir Crit Care Med 2002; 165: 277-304.

3 Thomeer M, Demedts M, Behr J, et al. Multidisciplinary interobserver agreement in the diagnosis of idiopathic pulmonary fibrosis. Eur Respir J 2008; 31: 585-591.

4 Flaherty KR, Andrei A-C, King TE Jr, et al. Idiopathic interstitial pneumonia: do community and academic physicians agree on diagnosis? Am J Respir Crit Care Med 2007; 175: 1054-1060.

5 Raghu G, Collard HR, Egan JJ, et al. An official ATS/ERS/JRS/ALAT statement: idiopathic pulmonary fibrosis: evidence-based guidelines for diagnosis and management. Am J Respir Crit Care Med 2011; 183: 788-824. Ryerson CJ, Collard HR. Update on the diagnosis and classification of ILD. Curr Opin Pulm Med 2013; 19: 453-459. Corte TJ, Copley SJ, Desai SR, et al. Significance of connective tissue disease features in idiopathic interstitial pneumonia. Eur Respir J 2012; 39: 661-668.

8 Flaherty KR, Thwaite EL, Kazerooni EA, et al. Radiological versus histological diagnosis in UIP and NSIP: survival implications. Thorax 2003; 58: 143-148.

9 Sverzellati N, Wells AU, Tomassetti S, et al. Biopsy-proved idiopathic pulmonary fibrosis: spectrum of nondiagnostic thin-section CT diagnoses. Radiology 2010; 254: 957-964.

10 Sumikawa H, Johkoh T, Colby TV, et al. Computed tomography findings in pathological usual interstitial pneumonia: relationship to survival. Am J Respir Crit Care Med 2008; 177: 433-439.

11 Travis WD, Hunninghake G, King TE Jr, et al. Idiopathic nonspecific interstitial pneumonia: report of an American Thoracic Society project. Am J Respir Crit Care Med 2008; 177: 1338-1347.

12 Akira M, Inoue Y, Arai T, et al. Long-term follow-up high-resolution CT findings in non-specific interstitial pneumonia. Thorax 2011; 66: 61-65.

13 Craig PJ, Wells AU, Doffman S, et al. Desquamative interstitial pneumonia, respiratory bronchiolitis and their relationship to smoking. Histopathology 2004; 45: 275-282. 
14 Bullard JE, Wert SE, Whitsett JA, et al. ABCA3 mutations associated with pediatric interstitial lung disease. Am J Respir Crit Care Med 2005; 172: 1026-1031.

15 Ichikado K, Suga M, Müller NL, et al. Acute interstitial pneumonia: comparison of high-resolution computed tomography findings between survivors and nonsurvivors. Am J Respir Crit Care Med 2002; 165: 1551-1556.

16 Rice AJ, Wells AU, Bouros D, et al. Terminal diffuse alveolar damage in relation to interstitial pneumonias: an autopsy study. Am J Clin Pathol 2003; 119: 709-714.

17 Song JW, Hong SB, Lim CM, et al. Acute exacerbation of idiopathic pulmonary fibrosis: incidence, risk factors and outcome. Eur Respir J 2011; 37: 356-363.

18 Agarwal R, Jindal SK. Acute exacerbation of idiopathic pulmonary fibrosis: a systematic review. Eur J Intern Med 2008; 19: 227-235.

19 Silva CIS, Müller NL, Fujimoto K, et al. Acute exacerbation of chronic interstitial pneumonia: high-resolution computed tomography and pathologic findings. J Thorac Imaging 2007; 22: 221-229.

20 Churg A, Müller NL, Silva CIS, et al. Acute exacerbation (acute lung injury of unknown cause) in UIP and other forms of fibrotic interstitial pneumonias. Am J Surg Pathol 2007; 31: 277-284.

21 Suda T, Kaida Y, Nakamura Y, et al. Acute exacerbation of interstitial pneumonia associated with collagen vascular diseases. Respir Med 2009; 103: 846-853.

22 Park IN, Kim DS, Shim TS, et al. Acute exacerbation of interstitial pneumonia other than idiopathic pulmonary fibrosis. Chest 2007; 132: 214-220.

23 Miyazaki Y, Tateishi T, Akashi T, et al. Clinical predictors and histologic appearance of acute exacerbations in chronic hypersensitivity pneumonitis. Chest 2008; 134: 1265-1270.

24 Cha SI, Fessler MB, Cool CD, et al. Lymphoid interstitial pneumonia: clinical features, associations and prognosis. Eur Respir J 2006; 28: 364-369.

25 Piciucchi S, Tomassetti S, Casoni G, et al. High resolution CT and histological findings in idiopathic pleuroparenchymal fibroelastosis: features and differential diagnosis. Respir Res 2011; 12: 111.

26 Margaritopoulos GA, Romagnoli M, Poletti V, et al. Recent advances in the pathogenesis and clinical evaluation of pulmonary fibrosis. Eur Respir Rev 2012; 21: 48-56.

27 Maher TM, Wells AU, Laurent GJ. Idiopathic pulmonary fibrosis: multiple causes and multiple mechanisms? Eur Respir J 2007; 30: 835-839.

28 Li X, Molina-Molina M, Abdul-Hafez A, et al. Extravascular sources of lung angiotensin peptide synthesis in idiopathic pulmonary fibrosis. Am J Physiol Lung Cell Mol Physiol 2006; 291: L887-L895.

29 Uhal BD, Dang M, Dang V, et al. Cell cycle dependence of ACE-2 explains downregulation in idiopathic pulmonary fibrosis. Eur Respir J 2013; 42: 198-210.

30 Chilosi M, Carloni A, Rossi A, et al. Premature lung aging and cellular senescence in the pathogenesis of idiopathic pulmonary fibrosis and COPD/emphysema. Transl Res 2013; 162: 156-173.

31 Chilosi M, Poletti V, Rossi A. The pathogenesis of COPD and IPF: distinct horns of the same devil? Respir Res 2012; 13: 23.

32 Chilosi M, Doglioni C, Murer B, et al. Epithelial stem cell exhaustion in the pathogenesis of idiopathic pulmonary fibrosis. Sarcoidosis Vasc Diffuse Lung Dis 2010; 27: 7-18.

33 Carloni A, Poletti V, Fermo L, et al. Heterogeneous distribution of mechanical stress in human lung: a mathematical approach to evaluate abnormal remodeling in IPF. J Theor Biol 2013; 332: 136-140.

34 Mendoza-Milla C, Valero Jiménez A, Rangel C, et al. Dehydroepiandrosterone has strong antifibrotic effects and is decreased in idiopathic pulmonary fibrosis. Eur Respir J 2013; 42: 1309-1321.

35 Maher TM. PROFILEing idiopathic pulmonary fibrosis: rethinking biomarker discovery. Eur Respir Rev 2013; 22: $148-152$.

36 Richeldi L, Collard HR, du Bois RM, et al. Mapping the future for pulmonary fibrosis: report from the 17th International Colloquium on Lung and Airway Fibrosis. Eur Respir J 2013; 42: 230-238.

37 Seibold MA, Wise AL, Speer MC, et al. A Common MUC5B promoter polymorphism and pulmonary fibrosis. N Engl J Med 2011; 364: 1503-1512.

38 Peljto AL, Zhang Y, Fingerlin TE, et al. Association between the MUC5B promoter polymorphism and survival in patients with idiopathic pulmonary fibrosis. JAMA 2013; 309: 2232-2239.

39 Stock CJ, Sato H, Fonseca C, et al. Mucin 5B promoter polymorphism is associated with idiopathic pulmonary fibrosis but not with development of lung fibrosis in systemic sclerosis or sarcoidosis. Thorax 2013; 68: 436-441.

40 Borie R, Crestani B, Dieude P, et al. The MUC5B variant is associated with idiopathic pulmonary fibrosis but not with systemic sclerosis interstitial lung disease in the European Caucasian population. PLoS One 2013; 8: e70621.

41 Yang IV, Coldren CD, Leach SM, et al. Expression of cilium-associated genes defines novel molecular subtypes of idiopathic pulmonary fibrosis. Thorax 2013; 68: 1114-1121.

42 Armanios MY, Chen JJ, Cogan JD, et al. Telomerase mutations in families with idiopathic pulmonary fibrosis. N Engl J Med 2007; 356: 1317-1326.

43 Tsakiri KD, Cronkhite JT, Kuan PJ, et al. Adult-onset pulmonary fibrosis caused by mutations in telomerase. Proc Natl Acad Sci USA 2007; 104: 7552-7557.

44 Fingerlin TE, Murphy E, Zhang W, et al. Genome-wide association study identifies multiple susceptibility loci for pulmonary fibrosis. Nat Genet 2013; 45: 613-620.

45 Noth I, Zhang Y, Ma S-F, et al. Genetic variants associated with idiopathic pulmonary fibrosis susceptibility and mortality: a genome-wide association study. Lancet 2013; 1: 309-317.

46 Yang IV, Luna LG, Cotter J, et al. The peripheral blood transcriptome identifies the presence and extent of disease in idiopathic pulmonary fibrosis. PLoS One 2012; 7: e37708.

47 Herazo-Maya JD, Noth I, Duncan SR, et al. Peripheral blood mononuclear cell gene expression profiles predict poor outcome in idiopathic pulmonary fibrosis. Sci Transl Med 2013; 5: 205ra136.

48 Yokoyama A, Kondo K, Nakajima M, et al. Prognostic value of circulating KL-6 in idiopathic pulmonary fibrosis. Respirology 2006; 11: 164-168.

49 Prasse A, Probst C, Bargagli E, et al. Serum CC-chemokine ligand 18 concentration predicts outcome in idiopathic pulmonary fibrosis. Am J Respir Crit Care Med 2009; 179: 717-723.

50 Greene KE, King TE Jr, Kuroki Y, et al. Serum surfactant proteins-A and -D as biomarkers in idiopathic pulmonary fibrosis. Eur Respir J 2002; 19: 439-446. 
51 Richards TJ, Kaminski N, Baribaud F, et al. Peripheral blood proteins predict mortality in idiopathic pulmonary fibrosis. Am J Respir Crit Care Med 2012; 185: 67-76.

52 Marchal-Sommé J, Uzunhan Y, Marchand-Adam S, et al. Dendritic cells accumulate in human fibrotic interstitial lung disease. Am J Respir Crit Care Med 2007; 176: 1007-1014.

53 Marchal-Sommé J, Uzunhan Y, Marchand-Adam S, et al. Cutting edge: nonproliferating mature immune cells form a novel type of organized lymphoid structure in idiopathic pulmonary fibrosis. J Immunol 2006; 176: 5735-5739.

54 Feghali-Bostwick CA, Tsai CG, Valentine VG, et al. Cellular and humoral autoreactivity in idiopathic pulmonary fibrosis. J Immunol 2007; 179: 2592-2599.

55 Taillé C, Grootenboer-Mignot S, Boursier C, et al. Identification of periplakin as a new target for autoreactivity in idiopathic pulmonary fibrosis. Am J Respir Crit Care Med 2011; 183: 759-766.

56 Kahloon RA, Xue J, Bhargava A, et al. Patients with idiopathic pulmonary fibrosis with antibodies to heat shock protein 70 have poor prognoses. Am J Respir Crit Care Med 2013; 187: 768-775.

57 Idiopathic Pulmonary Fibrosis Clinical Research Network, Raghu G, Anstrom KJ, et al. Prednisone, azathioprine, and N-acetylcysteine for pulmonary fibrosis. N Engl J Med 2012; 366: 1968-1977.

58 Wells AU, Behr J, Costabel U, et al. Triple therapy in idiopathic pulmonary fibrosis: an alarming press release. Eur Respir J 2012; 39: 805-806.

59 Hogaboam CM, Murray L, Martinez FJ. Epigenetic mechanisms through which Toll-like receptor-9 drives idiopathic pulmonary fibrosis progression. Proc Am Thorac Soc 2012; 9: 172-176.

60 Margaritopoulos GA, Antoniou KM, Karagiannis K, et al. Investigation of Toll-like receptors in the pathogenesis of fibrotic and granulomatous disorders: a bronchoalveolar lavage study. Fibrogenesis Tissue Repair 2010; 3: 20.

61 Lasithiotaki I, Antoniou KM, Vlahava VM, et al. Detection of herpes simplex virus type-1 in patients with fibrotic lung diseases. PLoS One 2011; 6: e27800.

62 Molyneaux PL, Maher TM. The role of infection in the pathogenesis of idiopathic pulmonary fibrosis. Eur Respir Rev 2013; 22: 376-381.

63 O’Dwyer DN, Armstrong ME, Trujillo G, et al. The Toll-like receptor 3 L412F polymorphism and disease progression in idiopathic pulmonary fibrosis. Am J Respir Crit Care Med 2013; 188: 1442-1450.

64 Caminati A, Cassandro R, Harari S. Pulmonary hypertension in chronic interstitial lung diseases. Eur Respir Rev 2013; 22: 292-301.

65 Judge EP, Fabre A, Adamali HI, et al. Acute exacerbations and pulmonary hypertension in advanced idiopathic pulmonary fibrosis. Eur Respir J 2012; 40: 93-100.

66 Patel NM, Kawut SM, Jelic S, et al. Pulmonary arteriole gene expression signature in idiopathic pulmonary fibrosis. Eur Respir J 2013; 41: 1324-1330.

67 King C, Nathan SD. Identification and treatment of comorbidities in idiopathic pulmonary fibrosis and other fibrotic lung diseases. Curr Opin Pulm Med 2013; 19: 466-473.

68 Kim DS. Acute exacerbations in patients with idiopathic pulmonary fibrosis. Respir Res 2013; 14: 86.

69 Allaix ME, Fisichella PM, Noth I, et al. Idiopathic pulmonary fibrosis and gastroesophageal reflux. Implications for treatment. J Gastrointest Surg 2014; 18: 100-105.

70 Savarino E, Carbone R, Marabotto E, et al. Gastro-oesophageal reflux and gastric aspiration in idiopathic pulmonary fibrosis patients. Eur Respir J 2013; 42: 1322-1331.

71 Cottin V, Nunes H, Brillet PY, et al. Combined pulmonary fibrosis and emphysema: a distinct underrecognised entity. Eur Respir J 2005; 26: 586-593.

72 Cottin V. The impact of emphysema in pulmonary fibrosis. Eur Respir Rev 2013; 22: 153-157.

73 Ryerson CJ, Hartman T, Elicker BM, et al. Clinical features and outcomes in combined pulmonary fibrosis and emphysema in idiopathic pulmonary fibrosis. Chest 2013; 144: 234-240.

74 Sode BF, Dahl M, Nielsen SF, et al. Venous thromboembolism and risk of idiopathic interstitial pneumonia: a nationwide study. Am J Respir Crit Care Med 2010; 181: 1085-1092.

75 Bargagli E, Madioni C, Bianchi N, et al. Serum analysis of coagulation factors in IPF and NSIP. Inflammation 2013 [In press DOI: 10.1007/s10753-013-9706-z].

76 Navaratnam V, Fogarty AW, McKeever T, et al. Presence of a prothrombotic state in people with idiopathic pulmonary fibrosis: a population-based case-control study. Thorax 2013 [In press DOI: 10.1136/thoraxjnl-2013203740].

77 Kubo H, Nakayama K, Yanai M, et al. Anticoagulant therapy for idiopathic pulmonary fibrosis. Chest 2005; 128 : $1475-1482$.

78 Noth I, Anstrom KJ, Calvert SB, et al. A placebo-controlled randomized trial of warfarin in idiopathic pulmonary fibrosis. Am J Respir Crit Care Med 2012; 186: 88-95.

79 Tomassetti S, Ruy JH, Gurioli Et Al C. The effect of anticoagulant therapy for idiopathic pulmonary fibrosis in real life practice. Sarcoidosis Vasc Diffuse Lung Dis 2013; 30: 121-127.

80 Coultas DB. Coagulation disorders and the IPF puzzle. Thorax 2013 [In press DOI: 10.1136/thoraxjnl-2013204245].

81 Smith JS, Gorbett D, Mueller J, et al. Pulmonary hypertension and idiopathic pulmonary fibrosis: a dastardly duo. Am I Med Sci 2013; 346: 221-225.

82 Kimura M, Taniguchi H, Kondoh Y, et al. Pulmonary hypertension as a prognostic indicator at the initial evaluation in idiopathic pulmonary fibrosis. Respiration 2013; 85: 456-463.

83 Gläser S, Obst A, Koch B, et al. Pulmonary hypertension in patients with idiopathic pulmonary fibrosis - the predictive value of exercise capacity and gas exchange efficiency. PLoS One 2013; 8: e65643.

84 Raghu G, Behr J, Brown KK, et al. Treatment of idiopathic pulmonary fibrosis with ambrisentan: a parallel, randomized trial. Ann Intern Med 2013; 158: 641-649.

85 Calabrese F, Kipar A, Lunardi F, et al. Herpes virus infection is associated with vascular remodeling and pulmonary hypertension in idiopathic pulmonary fibrosis. PLoS One 2013; 8: e55715.

86 Antoniou KM, Wells AU. Acute exacerbations of idiopathic pulmonary fibrosis. Respiration 2013; 86: 265-274.

87 Shulgina L, Cahn AP, Chilvers ER, et al. Treating idiopathic pulmonary fibrosis with the addition of cotrimoxazole: a randomised controlled trial. Thorax 2013; 68: 155-162.

88 Romagnoli M, Nannini C, Piciucchi S, et al. Idiopathic nonspecific interstitial pneumonia: an interstitial lung disease associated with autoimmune disorders? Eur Respir J 2011; 38: 384-391. 
89 Lee JS, Kim EJ, Lynch KL, et al. Prevalence and clinical significance of circulating autoantibodies in idiopathic pulmonary fibrosis. Respir Med 2013; 107: 249-255.

90 Pihtili A, Bingol Z, Kiyan E, et al. Obstructive sleep apnea is common in patients with interstitial lung disease. Sleep Breath 2013; 17: 1281-1288.

91 Mermigkis C, Bouloukaki I, Antoniou KM, et al. CPAP therapy in patients with idiopathic pulmonary fibrosis and obstructive sleep apnea: does it offer a better quality of life and sleep? Sleep Breath 2013; 17: 1137-1143.

92 Akhtar AA, Ali MA, Smith RP. Depression in patients with idiopathic pulmonary fibrosis. Chron Respir Dis 2013; 10: 127-133.

93 Bajwah S, Higginson IJ, Ross JR, et al. The palliative care needs for fibrotic interstitial lung disease: a qualitative study of patients, informal caregivers and health professionals. Palliat Med 2013; 27: 869-876.

94 Ravaglia C, Tomassetti S, Gurioli C, et al. Palliative medicine and end-of-life care in idiopathic pulmonary fibrosis. J Palliat Med 2013; 16: 339.

95 Behr J. Evidence-based treatment strategies in idiopathic pulmonary fibrosis. Eur Respir Rev 2013; 22: $163-168$.

96 Sakamoto S, Itoh T, Muramatsu Y, et al. Efficacy of pirfenidone in patients with advanced-stage idiopathic pulmonary fibrosis. Intern Med 2013; 52: 2495-2501.

97 Okuda R, Hagiwara E, Baba T, et al. Safety and efficacy of pirfenidone in idiopathic pulmonary fibrosis in clinical practice. Respir Med 2013; 107: 1431-1437.

98 Bonella F, Wessendorf TE, Costabel U. Clinical experience with pirfenidone for the treatment of idiopathic pulmonary fibrosis. Dtsch Med Wochenschr 2013; 138: 518-523.

99 Antoniou KM, Margaritopoulos GA, Siafakas NM. Pharmacological treatment of idiopathic pulmonary fibrosis: from the past to future. Eur Respir Rev 2013; 22: 281-291.

100 Vancheri C. Common pathways in idiopathic pulmonary fibrosis and cancer. Eur Respir Rev 2013; 22: $265-272$.

101 Tzouvelekis A, Paspaliaris V, Koliakos G, et al. A prospective, non-randomized, no placebo-controlled, phase Ib clinical trial to study the safety of the adipose derived stromal cells-stromal vascular fraction in idiopathic pulmonary fibrosis. J Transl Med 2013; 11: 171.

102 Antoniou KM, Margaritopoulos G, Economidou F, et al. Pivotal clinical dilemmas in collagen vascular diseases associated with interstitial lung involvement. Eur Respir J 2009; 33: 882-896.

103 Lindahl GE, Stock CJ, Shi-Wen X, et al. Microarray profiling reveals suppressed interferon stimulated gene program in fibroblasts from scleroderma-associated interstitial lung disease. Respir Res 2013; 14: 80.

104 Vancheri C, du Bois RM. A progression-free end-point for idiopathic pulmonary fibrosis trials: lessons from cancer. Eur Respir J 2013; 41: 262-269.

105 Goh NS, Desai SR, Veeraraghavan S, et al. Interstitial lung disease in systemic sclerosis: a simple staging system. Am J Respir Crit Care Med 2008; 177: 1248-1254.

106 Goh NS, Desai SR, Anagnostopoulos C, et al. Increased epithelial permeability in pulmonary fibrosis in relation to disease progression. Eur Respir J 2011; 38: 184-190.

107 Tiev KP, Hua-Huy T, Kettaneh A, et al. Serum CC chemokine ligand-18 predicts lung disease worsening in systemic sclerosis. Eur Respir J 2011; 38: 1355-1360.

108 Beirne P, Pantelidis P, Charles P, et al. Multiplex immune serum biomarker profiling in sarcoidosis and systemic sclerosis. Eur Respir J 2009; 34: 1376-1382.

109 De Lauretis A, Sestini P, Pantelidis P, et al. Serum interleukin 6 is predictive of early functional decline and mortality in interstitial lung disease associated with systemic sclerosis. J Rheumatol 2013; 40: 435-446.

110 Olson AL, Swigris JJ, Sprunger DB, et al. Rheumatoid arthritis-interstitial lung disease-associated mortality. Am J Respir Crit Care Med 2011; 183: 372-378.

111 Kim EJ, Elicker BM, Maldonado F, et al. Usual interstitial pneumonia in rheumatoid arthritis-associated interstitial lung disease. Eur Respir J 2010; 35: 1322-1328.

112 Harlow L, Rosas IO, Gochuico BR, et al. Identification of citrullinated hsp90 isoforms as novel autoantigens in rheumatoid arthritis-associated interstitial lung disease. Arthritis Rheum 2013; 65: 869-879.

113 Giles JT, Danoff SK, Sokolove J, et al. Association of fine specificity and repertoire expansion of anticitrullinated peptide antibodies with rheumatoid arthritis associated interstitial lung disease. Ann Rheum Dis 2013 [In press DOI: 10.1136/annrheumdis-2012-203160].

114 Antoniou KM, Walsh SL, Hansell DM, et al. Smoking-related emphysema is associated with idiopathic pulmonary fibrosis and rheumatoid lung. Respirology 2013; 18: 1191-1196.

115 Fischer A, Brown KK, Du Bois RM, et al. Mycophenolate mofetil improves lung function in connective tissue disease-associated interstitial lung disease. J Rheumatol 2013; 40: 640-646.

116 Keir GJ, Maher TM, Ming D, et al. Rituximab in severe, treatment-refractory interstitial lung disease. Respirology 2013 [In press DOI: 10.1111/resp.12214].

117 Zhou T, Zhang W, Sweiss NJ, et al. Peripheral blood gene expression as a novel genomic biomarker in complicated sarcoidosis. PLoS One 2012; 7: e44818.

118 Grunewald J, Brynedal B, Darlington P, et al. Different HLA-DRB1 allele distributions in distinct clinical subgroups of sarcoidosis patients. Respir Res 2010; 11: 25.

119 Grunewald J, Eklund A, Olerup O. Human leukocyte antigen class I alleles and the disease course in sarcoidosis patients. Am J Respir Crit Care Med 2004; 169: 696-702.

120 Rossman MD, Thompson B, Frederick M, et al. HLA-DRB1*1101: a significant risk factor for sarcoidosis in blacks and whites. Am J Hum Genet 2003; 73: 720-735.

121 Valentonyte R, Hampe J, Huse K, et al. Sarcoidosis is associated with a truncating splice site mutation in BTNL2. Nat Genet 2005; 37: 357-364.

122 Panselinas E, Judson MA. Acute pulmonary exacerbations of sarcoidosis. Chest 2012; 142: 827-836.

123 Baughman RP, Lower EE. Frequency of acute worsening events in fibrotic pulmonary sarcoidosis patients. Respir Med 2013; 107: 2009-2013.

124 Culver DA, Costabel U. EBUS-TBNA for the diagnosis of sarcoidosis: is it the only game in town? J Bronchology Interv Pulmonol 2013; 20: 195-197.

125 Wong M, Yasufuku K, Nakajima T, et al. Endobronchial ultrasound: new insight for the diagnosis of sarcoidosis. Eur Respir J 2007; 29: 1182-1186. 
126 Tremblay A, Stather DR, Maceachern P, et al. A randomized controlled trial of standard vs endobronchial ultrasonography-guided transbronchial needle aspiration in patients with suspected sarcoidosis. Chest 2009; 136: 340-346.

127 von Bartheld MB, Dekkers OM, Szlubowski A, et al. Endosonography vs conventional bronchoscopy for the diagnosis of sarcoidosis: the GRANULOMA randomized clinical trial. JAMA 2013; 309: 2457-2464.

128 Agarwal R, Aggarwal AN, Gupta D. Efficacy and safety of conventional transbronchial needle aspiration in sarcoidosis: a systematic review and meta-analysis. Respir Care 2013; 58: 683-693.

129 Patel AS, Siegert RJ, Creamer D, et al. The development and validation of the King's Sarcoidosis Questionnaire for the assessment of health status. Thorax 2013; 68: 57-65.

130 Cox CE, Donohue JF, Brown CD, et al. The Sarcoidosis Health Questionnaire: a new measure of health-related quality of life. Am J Respir Crit Care Med 2003; 168: 323-329.

131 Costabel U, Ohshimo S, Guzman J. Diagnosis of sarcoidosis. Curr Opin Pulm Med 2008; 14: 455-461.

132 Chapelon-Abric C. Cardiac sarcoidosis. Curr Opin Pulm Med 2013; 19: 493-502.

133 Schuller JL, Zipse M, Crawford T, et al. Implantable cardioverter defibrillator therapy in patients with cardiac sarcoidosis. J Cardiovasc Electrophysiol 2012; 23: 925-929.

134 Baughman RP, Nunes H. Therapy for sarcoidosis: evidence-based recommendations. Expert Rev Clin Immunol 2012; 8: 95-103.

135 Baughman RP, Nunes H, Sweiss NJ, et al. Established and experimental medical therapy of pulmonary sarcoidosis. Eur Respir J 2013; 41: 1424-1438.

136 Vorselaars AD, Wuyts WA, Vorselaars VM, et al. Methotrexate vs azathioprine in second-line therapy of sarcoidosis. Chest 2013; 144: 805-812.

137 Baughman RP, Winget DB, Lower EE. Methotrexate is steroid sparing in acute sarcoidosis: results of a double blind, randomized trial. Sarcoidosis Vasc Diffuse Lung Dis 2000; 17: 60-66.

138 Lower EE, Baughman RP. Prolonged use of methotrexate for sarcoidosis. Arch Intern Med 1995; 155: 846-851. 